\section{A defense of the two-component model of visual facilitation in auditory localization}

\author{
BRIAN R. SHELTON \\ University of Western Ontario, London, Ontario, Canada
}

A paper published by Campbell Searle and myself (Shelton \& Searle, 1980) has come under criticism in a recent article in this journal (Mastroianni, 1982). I would like to respond to some of the issues raised by those comments, and to examine the utility of the experiment Mastroianni conducted to resolve the apparent conflict.

Our original report took the form of two distinct experiments. The first dealt with the visual facilitation of auditory localization judgments in an absolute identification paradigm. Localization abilities of sighted and blindfolded observers were compared with horizontal speaker arrays located directly in front, to the side, and behind the head of the subject, and with a span of speakers in the vertical plane. The effects of blindfolding the observers depended on the orientation of the speaker array, but there was as much as a sevenfold increase in average error in some conditions.

In the second experiment, we presented a single 200 -msec burst of white noise as the localization stimulus. The visual environment was controlled by providing no room illumination and marking specific locations with light-emitting diodes. Subjects were presented with a noise burst from one of two speakers, and required to identify the sound as coming from the left or right source. The subjects made their judgments in total darkness or in the presence of a pair of lights. We examined a number of temporal and spatial parameters of visual stimulation to determine which configurations influenced localization accuracy relative to performance in total darkness.

We found only two conditions which facilitated localization judgments. Closely spaced lights positioned between the two alternatives improved discrimination if they were presented $800 \mathrm{msec}$ before the localization stimulus. Likewise, lights that marked the exact position of the speakers and remained on $800 \mathrm{msec}$ after the localization stimulus ended also facilitated performance. These two conditions seem to correspond to two ideas discussed in the localization literature to explain visual facil-

I would like to express my thanks to Steve Lupker for his helpful comments on this manuscript. My mailing address is: Psychology Department, University of Western Ontario, London, Ontario N6A 5C2, Canada. itation, that is, the frame-of-reference hypothesis and the memory-facilitation hypothesis. Our review of the literature indicated that support of the frame-of-reference hypothesis had been obtained with paradigms in which localization sources remained on while observers made their judgments, and that support for the memory-facilitation position had been obtained with very brief, transient stimuli. We therefore made the suggestion that these two processes were both involved in the visual facilitation of auditory localization, and that the two specific effects we had observed reflected these processes. We argued that the demands of the task would determine the exact function served by vision in facilitating auditory localization.

Mastroianni's criticism of the first experiment involves the fact that the sighted observers were allowed to see the actual speaker positions during testing, rather than having a blank visual field. Our critic claims that the fact that the speakers were visible to the sighted subjects makes the outcome of the experiment unsurprising, and, presumably, uninteresting. I, for one, do not know the body of knowledge which allows Mastroianni to be unimpressed by the experimental demonstration we provided and yet still be interested in the phenomenon of visual facilitation. Searle, Braida, Davis, and Colburn (1976) reviewed over 30 localization experiments which used an absolute identification paradigm similar to the one we employed. Since we did show a substantial visual facilitation of localization judgments in a reasonably standard procedure, I fail to see the validity of Mastroianni's complaint.

There is an implicit contradiction between this same criticism and the one-component model of visual facilitation that Mastroianni is defending. That is, Mastroianni's criticism implies that the visual facilitation reported in our absolute identification experiment is somehow different from the visual facilitation reported in procedures that hide the speaker positions from the subjects' view. This opinion is plausible under the two-component model Searle and I discussed, but it is nonsensical with a one-component model. How can there be more than one type of visual facilitation if there is only one process involved? It is consistent with the two-component model to suggest that the results of our initial experiment were dominated by one component of the process, and in fact I tend to agree with Mastroianni's assessment. I do not see, however, how a one-component model can be defended by such a suggestion, or how the component process involved in our first experiment can be arbitrarily designated as uninteresting. 
The second experiment we conducted was criticized for the interpretation we made of our data. Mastroianni feels that there is a contradiction between our argument and previous findings by Jones (1975) and Warren (1970) which makes our interpretation untenable. The contradiction Mastroianni perceives is that we accept the Warren experiment as a demonstration of a frame-of-reference effect, and the Jones experiment as a demonstration of memory facilitation-even though both effects were demonstrated under full room illumination with no explicit speaker markers. Our position suggests that closely spaced lights that precede the sound source serve the same function as overall illumination in the Warren experiment, and that cospatial lights that remain on after the sound serve the same function as general room illumination in the Jones experiment.

Mastroianni's error is that he has ignored the crucial difference between the experiments of Warren and Jones. Warren's auditory stimuli were of long duration, and subjects made their judgments with the auditory stimulus present: There is no memory component to the Warren experiment. Jones, on the other hand, used brief auditory signals in a signaldetection paradigm. Since the acoustic stimulation was brief, the subjects made their responses after the signals terminated, and memory is an important aspect to this task. The response characteristics also differed radically. Warren required a pointing response in most of his studies, whereas Jones used a verbal identification response. A pointing response might very well utilize a visual frame of reference, because of the high degree of visual guidance normally afforded a pointing response. The demands of the tasks differ so radically that it is certainly conceivable that the same visual stimulation could serve very different functions in the two experiments. In the Warren task, the demand for a frame of reference was optimized, and in the Jones experiment, the need for an accurate spatial memory was stressed, and it seems quite possible that vision served these functions.

It is quite probable, then, that different processes are involved in the Warren and Jones experiments. It should be noted that even if we were to accept the situation as Mastroianni presents it, that identical stimulus conditions have provided support for opponent theories, the observed data would cause problems for any theoretical position. As such, even if the criticism was presented in good faith, it could not be used to support one theoretical position over another.

The failure of Mastroianni to grasp the importance of the acoustic parameters in determining the function served by vision is reflected in the experiment he chose to perform to address the issue. In order to examine the question of the influence of vision on localization, Mastroianni offered a replication of Warren's experiments. The basic findings held, and Mastroianni concluded that the results tended to support the frame-of-reference hypothesis. In terms of the two-component model, this is the expected outcome because, just as the original Warren study, a pointing response was used and there was no memory load. It is unreasonable to expect anyone to change his view of visual facilitation, given the evidence Mastroianni provides, and I reiterate the opinion that both a frame-of-reference and a memory-facilitation effect can be demonstrated, given the proper stimulus configuration. Mastroianni's result seems to be yet another demonstration of this fact.

\section{REFERENCES}

JoNes, B. Visual facilitation of auditory localization in school children: A signal detection analysis. Perception \& Psychophysics, 1975, 17, 217-220.

Mastrolanni, G. $R$. The influence of eye movements and illumination on auditory localization. Perception \& Psychophysics, 1982, 31, 581-584.

Searle, C. L., Braida, L. D., Davis, M. F., \& Colburn, H. S. Model for auditory localization. Journal of the Acoustical Society of America, 1976, 60, 1164-1175.

Shelton, B. R., \& Searle, C. L. The influence of vision on the absolute identification of sound-source position. Perception \& Psychophysics, 1980, 28, 589-596.

WARREN, D. H. Intermodal interactions in spatial localization. Cognitive Psychology, 1970, 1, 114-133.

(Manuscript received January 10, 1983; accepted for publication January 23, 1983.) 